\title{
Microbiological and genotypic factors affecting mortality in methicillin-resistant Staphylococcus aureus bacteremia
}

\author{
Eun-Jeong Joo
}

Division of Infectious Diseases, Department of Internal Medicine, Kangbuk Samsung Hospital, Sungkyunkwan University School of Medicine, Seoul, Korea

Received: December 4, 2018 Accepted: December 11, 2018

\section{Correspondence to} Eun-Jeong Joo, M.D.

Division of Infectious Diseases, Department of Internal Medicine, Kangbuk Samsung Hospital, Sungkyunkwan University School of Medicine, 29 Saemunan-ro, Jongno-gu, Seoul 03181, Korea

Tel: +82-2-2001-8533

Fax: +82-2-2001-1596

E-mail:iamjoo@skku.edu

\section{See Article on Page 184-194}

Staphylococcus aureus causes a wide range of skin, soft tissue, bone, and joint infections and invasive infections associated with indwelling catheters or prosthetic devices, bacteremia, endocarditis, and pneumonia. Methicillin-resistant S. aureus (MRSA) is a leading cause of nosocomial bacteremia and is associated with increased morbidity and mortality compared to susceptible isolates in $S$. aureus bacteremia (SAB) [1]. MRSA features associated with poor clinical outcomes include the vancomycin minimum inhibitory concentration (MIC), vancomycin heteroresistant phenotype, expression of virulence toxins, and accessor gene regulator (agr) functionality. In a prospective cohort of Korean patients with MRSA bacteremia, Kim et al. [2] found that the severity of illness was significantly associated with early mortality. Interestingly, a higher vancomycin MIC was inversely associated with severe sepsis or septic shock [2].

It is controversial whether SAB caused by an isolate with reduced vancomycin susceptibility is associated with poorer outcomes than that caused by isolates highly susceptible to vancomycin [3-5]. Paradoxically, a higher risk of mortality has been seen with an E-test for an MIC $<1 \mu \mathrm{g} / \mathrm{mL}$ than for an MIC $>1.5 \mu \mathrm{g} /$
$\mathrm{mL}$ [6]. In a study of the fitness of MRSA and glycopeptide-intermediate $S$. aureus strains, a reduction in the growth rate was observed in the two strain types [7]. $S$. aureus strains with high vancomycin MICs might be less virulent than strains with low vancomycin MICs. Therefore, an increase in vancomycin MIC within the susceptible range might be a surrogate marker for intrinsic microbiological traits and not associated with worse clinical outcomes [8].

Another important issue is whether the virulence factor of MRSA strains increases mortality in MRSA infections, especially by community-genotype strains. The USA3oo clone is most often associated with community-acquired (CA) skin and soft tissue infections and belongs to multilocus sequence type (ST) 8. This isolate characteristically contains the SCCmec type IV element and a phage carrying the genes encoding Panton-Valentine leucocidin (PVL), which involves in interactions between the pathogen and neutrophils and contributes to the establishment of $S$. aureus infection and determines the increased virulence of CA-MRSA compared to hospital-acquired MRSA. In South Korea, a PVL-negative CA-MRSA clone, ST72-SCCmec type IV, is widespread in both the community and hospitals [9,10]. Despite heightened 
concerns that ST72 MRSA may cause more severe disease and have poorer clinical outcomes than the hospital-genotype strain ST5-SCCmec type II, the clinical impact of $\mathrm{ST}_{72}$ MRSA on mortality in patients with MRSA bacteremia indicates that it has relatively lower virulence [11]. Although it is still not clear what virulence factor has facilitated the successful settlement of ST72 MRSA in healthcare settings, the introduction of $\mathrm{ST}_{72}$ MRSA into Korean hospitals seems to have fewer adverse effects with regard to disease distribution and all-cause mortality in patients with nosocomial MRSA infections [9].

In a prospective study of the risk factors for persistent MRSA bacteremia in the Korean population [12], no significant differences in the vancomycin MIC distribution or genotypic distribution of putative virulence genes and agr dysfunction were found in MRSA isolates between groups with persistent and resolving bacteremia. Clinical features such as the severity of illness, site of infection, and control of infection sources may have a greater effect on persistent MRSA bacteremia and risk for death from MRSA bacteremia, rather than the microbiological and genotypic features of the MRSA strain. Given the limitations of available treatments, especially with regard to vancomycin, which has a relatively slow onset of bactericidal activity and poor penetration of infected tissues, an early imaging approach that identifies hidden sources of infection and removes them by drainage and debridement should be implemented as a treatment strategy in MRSA bacteremia. Timely and appropriate vancomycin dosing may play a significant role in clinical and microbiological success.

\section{Conflict of interest}

No potential conflict of interest relevant to this article was reported.

\section{REFERENCES}

1. Joo EJ, Park DA, Kang CI, et al. Reevaluation of the impact of methicillin-resistance on outcomes in patients with Staphylococcus aureus bacteremia and endocarditis. Korean J Intern Med 2018 Jan 20 [Epub]. https://doi. org/10.3904/kjim.2017.098.

2. Kim T, Chong YP, Park KH, et al. Clinical and microbiological factors associated with early patient mortality from methicillin-resistant Staphylococcus aureus bacteremia. Korean J Intern Med 2018;34:184-194.

3. Soriano A, Marco F, Martinez JA, et al. Influence of vancomycin minimum inhibitory concentration on the treatment of methicillin-resistant Staphylococcus aureus bacteremia. Clin Infect Dis 2008;46:193-200.

4. van Hal SJ, Lodise TP, Paterson DL. The clinical significance of vancomycin minimum inhibitory concentration in Staphylococcus aureus infections: a systematic review and meta-analysis. Clin Infect Dis 2012;54:755-771.

5. Kalil AC, Van Schooneveld TC, Fey PD, Rupp ME. Association between vancomycin minimum inhibitory concentration and mortality among patients with Staphylococcus aureus bloodstream infections: a systematic review and meta-analysis. JAMA 2014;312:1552-1564.

6. Price J, Atkinson S, Llewelyn M, Paul J. Paradoxical relationship between the clinical outcome of Staphylococcus aureus bacteremia and the minimum inhibitory concentration of vancomycin. Clin Infect Dis 2009;48:997-998.

7. Noto MJ, Fox PM, Archer GL. Spontaneous deletion of the methicillin resistance determinant, mecA, partially compensates for the fitness cost associated with high-level vancomycin resistance in Staphylococcus aureus. Antimicrob Agents Chemother 2008;52:1221-1229.

8. Cameron DR, Ward DV, Kostoulias X, et al. Serine/threonine phosphatase Stpı contributes to reduced susceptibility to vancomycin and virulence in Staphylococcus aureus. J Infect Dis 2012;205:1677-1687.

9. Joo EJ, Chung DR, Kim SH, et al. Emergence of community-genotype methicillin-resistant Staphylococcus aureus in Korean hospitals: clinical characteristics of nosocomial infections by community-genotype strain. Infect Chemother 2017;49:109-116.

10. Joo EJ, Chung DR, Ha YE, et al. Community-associated Panton-Valentine leukocidin-negative meticillin-resistant Staphylococcus aureus clone (ST72-MRSA-IV) causing healthcare-associated pneumonia and surgical site infection in Korea. J Hosp Infect 2012;81:149-155.

11. Park KH, Chong YP, Kim SH, et al. Community-associated MRSA strain ST72-SCCmecIV causing bloodstream infections: clinical outcomes and bacterial virulence factors. J Antimicrob Chemother 2015;70:1185-1192.

12. Chong YP, Park SJ, Kim HS, et al. Persistent Staphylococcus aureus bacteremia: a prospective analysis of risk factors, outcomes, and microbiologic and genotypic characteristics of isolates. Medicine (Baltimore) 2013;92:98-108. 